\title{
Compliant gait assistance triggered by user intention*
}

\author{
Vijaykumar Rajasekaran, Joan Aranda, Member IEEE, and Alicia Casals, Senior Member IEEE
}

\begin{abstract}
An automatic gait initialization strategy based on user intention sensing in the context of rehabilitation with a lower-limb wearable robot is proposed and evaluated. The proposed strategy involves monitoring the human-orthosis interaction torques and initial position deviation to determine the gait initiation instant and to modify orthosis operation for gait assistance, when needed. During gait, the compliant control algorithm relies on the adaptation of the joints' stiffness in function of their interaction torques and their deviation from the desired trajectories, while maintaining the dynamic stability. As a reference input, the average of a set of recorded gaits obtained from healthy subjects is used. The algorithm has been tested with five healthy subjects showing its efficient behavior in initiating the gait and maintaining the equilibrium while walking in presence of external forces. The work is performed as a preliminary study to assist patients suffering from incomplete Spinal cord injury and Stroke.
\end{abstract}

Keywords - Exoskeleton, wearable robot, gait initiation, adaptive control, gait assistance, rehabilitation.

\section{INTRODUCTION}

Gait assistance using wearable robots is challenging in determining the suitable assistance for dynamic stability, considering the ground reaction forces acting on them. Several wearable robots have succeeded in providing dynamic stability, such as BLEEX [1], XPED2 [2], Ekso (earlier eLegs) [3], Rex (Rex Bionics) and Re-Walk [4]. These exoskeletons have proven to be efficient in providing assistance on a passive range of motion and using complex systems. However, human centered rehabilitation is essential for ensuring the user involvement in a therapy. Several human centered strategies, such as patient cooperative and support motor function assessment, oriented to the development of robot behaviors have been widely studied [5]. These strategies support the assist-as-needed concept by determining the level of robotic assistance provided to the user.

This paper presents an assistive control strategy for a wearable robot to perform user-triggered gait initiation and convenient assistance. The gait initiation approach ensures the user involvement in the therapy and their motivation in performing the task. The user motivation is necessary for an assistive rehabilitation procedure to avoid slacking. Moreover, this modifies the role of the patient in the therapy from object to subject, as the patient takes volitional control of the evolution of the gait.

The detection of the best instant for gait initiation and termination has been studied by many researchers in order to

*This research is supported by HYPER project (Hybrid Neuroprosthetic and Neurorobotic devices for functional compensation and rehabilitation of motor disorders), grant CSD2009-00067 CONSOLIDER INGENIO 2010 from MINECO (Spanish Ministry for Science and Education))

V. Rajasekaran, J. Aranda and A. Casals are associated with the Institute for Bioengineering of Catalunya and Universitat Politècnica de Catalunya, Barcelona-Tech, Spain. (Corresponding author's e-mail: rajasekaran.vijaykumar@gmail.com). develop a volitional based robotic rehabilitation [6, 7]. One of the widely used approaches for monitoring the human intention relies on the use of brain machine interfaces such as in XoR [8]. These systems are efficient in monitoring the user intentions mainly, because a real displacement of the joint position is not needed to initiate the gait. Instead, gait initiation in MINDWALKER is based on the displacement of the CoM (center of mass) which is calculated heuristically [9]. HAL (Hybrid Assistive limb), a light weight powered exoskeleton suit, is efficient in adapting to the user movements by sensing the muscle synergies. HAL uses myoelectric signals to measure the muscle forces and to support voluntary motion of the patient [10].

Referring to the gait, assistance must be dynamically adapted to the patient's needs and thus, it is essential to develop a personalized assistance in function of the user intentions and movements, and also adjust the level of assistance with regard to the evolution of the therapy. The use of a predefined walking trajectory pattern as the only target to be satisfied imposes a complete assistance which might induce slacking and harm the patient. Thus, it is necessary to design a hybrid combination of force-position control.

The goal of this work is to develop an efficient control model for a low-cost wearable robot and to validate the assistive behavior of the robot for patients with neurological disorders. A hybrid position and interaction torques based control strategy is presented to continuously adapt the user movements to the desired gait pattern in real time. This real time adaptation also ensures synchronization among the joint trajectories to maintain dynamic stability.

\section{HUMAN CENTERED GAIT ASSISTANCE}

The human-orthosis interaction torques are essential in defining the dynamic analysis of a human centered control strategy. Hence, the mathematical model for the dynamic analysis of an exoskeleton can be represented as

$$
M_{\text {ort }}(q) \ddot{q}+C_{\text {ort }}(q, \dot{q})+G_{\text {ort }}(q)=\tau_{a}+\tau_{\text {pat }}+\tau_{d}
$$

where $q, \dot{q}, \ddot{q}$ are the vectors of joint positions, velocities and accelerations respectively. $M_{\text {ort }}(q)$ is the inertia matrix, $C_{\text {ort }}(q, \dot{q})$ is the centrifugal and Coriolis vector and $G_{\text {ort }}(q)$ represents the gravitational torques. $\tau_{a}$ and $\tau_{\text {pat }}$ are the orthosis and patient torques respectively and $\tau_{d}$ correspond to the external disturbances acting on the subject. These actuator and patient torques are influenced by the human-orthosis interaction, while the external disturbances can be due to any assistive or external sources which can affect the dynamic stability of the robot. In the present work, $\tau_{\text {pat }}$ is used for both gait initiation and to determine the level of assistance to be exerted by the orthosis.

\section{A. Gait Initiation}

The gait initiation can be defined as the time $t$ when the user intends to perform their movement. Both the joint 
position deviation and torques are used in our approach to identify gait initialization, because torques by themselves can be misinterpreted with patient tremors.

Thus, the proposed gait initiation strategy considers the joint positions and the human-orthosis interaction torques of both legs. Hence this strategy permits the users to initiate the therapy with whichever leg they are more comfortable. The initialization condition is to detect some predefined deviation and torque in one leg while maintaining the other constant.

This can be represented as,

$$
G_{\text {init }}=\operatorname{Leg}_{x}, x \in[R, L]
$$

where, $G_{\text {init }}$ is the gait initiation trigger obtained from the user for $\operatorname{Leg}_{x}$.

\section{B. Gait Assistance}

In an exoskeleton each joint actuator works in collaboration with the patient. The actuator torque can be modified by varying the joint stiffness parameter, which invariably determines the degree of control transferred from the orthosis to the human or vice versa. Such an impedance control scheme has been widely used for its compliant behavior [11], which results in an adaptive walking pattern and a more natural interaction between patient and orthosis. Thus the impedance control can be determined as

$$
F=M a+C v+K \cdot\left(\theta_{\text {ref }}-\theta_{\text {act }}\right)
$$

where, $\theta_{\text {ref }}$ and $\theta_{\text {act }}$ are the reference and actual joint positions respectively, $\mathrm{K}$ is the stiffness parameter of the joint and $\mathrm{F}$ represents the applied force to the joint. $\mathrm{M}$ represents the mass, $\mathrm{C}$ is the damping constant and $a$ and $v$ represent the acceleration and velocity of the robot. The input sample rate is maintained constant and the damping coefficient is kept small, therefore the velocity of the orthosis is not modified by the user. Hence the force equation, influenced by the position error, is modified as

$$
F=K\left(\theta_{\text {ref }}-\theta_{\text {act }}\right)
$$

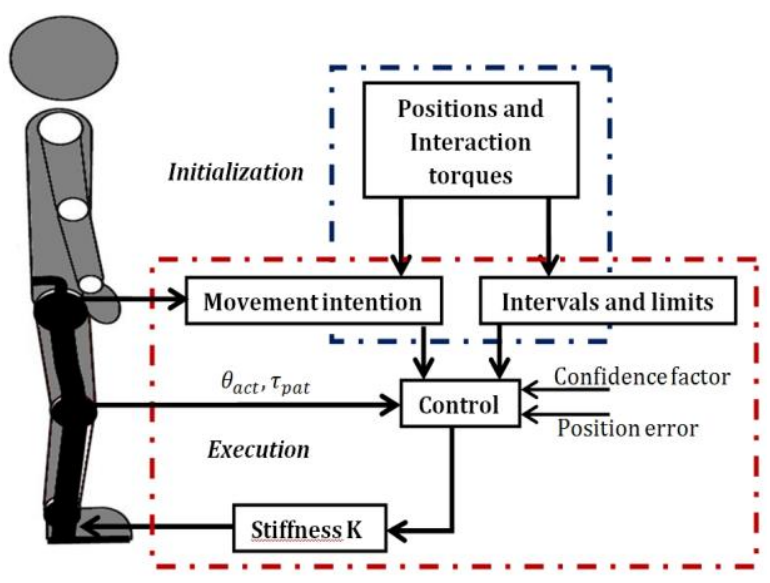

Figure 1. Schematic representation of the user-intention based adaptive control strategv

In this paper, the modification of the $K$ value is performed dynamically based on the performance of the user and the level of assistance to be exerted by the orthosis.

$$
\begin{aligned}
& K_{t+1}=K_{t} \pm \Delta K \\
& \Delta K=\left|\left(\frac{\theta_{r e f}-\theta_{a c t}}{s *\left(\tau_{\text {pat }}\right)}\right)\right|
\end{aligned}
$$

where, $\tau_{\text {pat }}$ is the human-orthosis interaction torques and $s$ is a confidence factor which is used to determine the stiffness to be applied at time $t+1$. The confidence factor is a variable parameter which shall be defined by the therapist as a function of the patient's performance. This confidence factor can be varied according to the progress of the user. A low confidence factor means that the assistance should be provided partially or completely to the procedure and a higher confidence factor indicates that the subject is capable of walking with very little assistance or without it.

The stiffness variation module (control box in fig. 1) is responsible for incrementing, decrementing or maintaining the stiffness parameter of each joint. The following are the parameters involved in defining this stiffness variation:

$$
\begin{aligned}
& \text { Algorithm for Gait assistance } \\
& \text { Case } \theta_{e}>\theta_{e} T h_{u p} \\
& \qquad K=K+\Delta K \\
& \text { Case } \theta_{e}<\theta_{e} T h_{l o} \& \& \tau_{p a t} T h_{l o}<\tau_{\text {pat }}<\tau_{\text {pat }} T h_{u p} \\
& K=K-\Delta K \\
& \text { Else } \\
& \quad \text { Maintain } K
\end{aligned}
$$

\section{Maintain $K$}

$\theta_{e}$ - Position error (deg)

$\theta_{e} T h_{u p}$ - Upper threshold of position error (deg)

$\theta_{e} T h_{l o}-$ Lower threshold of position error (deg)

$\tau_{\text {pat }}$ - Human-orthosis interaction torques (Nm)

$\tau_{\text {pat }} T h_{l o}$ - Lower threshold of interaction torques (Nm)

$\tau_{\text {pat }} T h_{u p}$ - Upper threshold of interaction torques (Nm)

$\Delta K$ - Stiffness variation $(\mathrm{N} / \mathrm{m})$

\section{EXPERIMENTAL PROCEDURE}

The experimentation consists of two phases: initialization and execution. The initialization phase involves monitoring the interaction torques and joint positions with no-assistance provided by the orthosis in order to permit the therapist to define the confidence factor $\mathrm{s}$. The necessary information, for the control model, to adjust the thresholds used in gait initialization and gait assistance is obtained in the initialization phase. In the execution phase, the changes in movement and interaction torques are used as a trigger for gait initiation. During gait assistance, trajectory deviation and interaction torques, limited by the confidence factor, are used to determine the time intervals for stiffness variation. Both these phases are performed and evaluated using a lower-limb exoskeleton, $\mathrm{H} 1$.

$\mathrm{H} 1$ is a 6 DoF (Degree of Freedom) wearable lower limb orthosis with an anthropomorphic configuration to assist individuals with incomplete Spinal cord injury (iSCI) or Stroke. The exoskeleton has been built within the framework of the Hyper* project. H1 has three joints for each leg: hip, knee and ankle, each joint is powered by a DC motor coupled with a harmonic drive gear. The exoskeleton is equipped with potentiometers and strain gauges to measure the joint angles and human-orthosis interaction torques on the links respectively. A detailed description about the exoskeleton structure and hardware is detailed in [12].

\section{A. Initialization}

Initially the walking pattern is tested on subjects applying a low stiffness value $(20 \mathrm{~N} / \mathrm{m})$. This phase is necessary to 
obtain the pattern of interaction torques and to adapt themselves to the orthosis. This initialization also determines the minimum interaction torque observed in the users along with the deviation in position.

The setup includes the generation of a reference gait pattern performed by healthy users and optimized after some repetitions of gait cycles. The values of stiffness and confidence factor are defined based on the subject's health condition. Since the strategy is tested with healthy individuals, the initial stiffness and confidence factor is assumed to be $50 \mathrm{~N} / \mathrm{m}$ and 0.9 respectively. In a first set of trials, for each subject the maximum interaction torques are obtained by applying a low stiffness value at each joint. From these maxima, we extract the upper and lower thresholds of interaction torques to dynamically define the operation mode. These thresholds are obtained by multiplying these maxima interaction torques by the confidence factor $(s)$.

\section{B. Protocol}

This study is performed as a preliminary evaluation of the control strategy, prior to clinical trials with patients suffering from iSCI and stroke. Thus, following the rehabilitation protocol an intermediate pause must be included between consecutive trials to ensure the active participation of the user, comfort in walking and to avoid muscle fatigue. For gait initiation, it is necessary to provide a time period long enough to allow identifying the user intention after recovering from fatigue.

In case of iSCI patients, therapy procedures consider a pause of 1minute at the end of every 2-3 minutes of walking, considering their fatigue. Since this study involves healthy subjects the experimental time can be higher and the pause time can be increased. For evaluating the gait initiation algorithm, an interval of 10-20 seconds is introduced at the beginning of each trial. An auditory cue is provided to notify the subject to initiate the movement. Similarly for evaluating the gait assistance strategy it is necessary to analyze the walking for a continuous span of time. Hence an intermediate pause time of 1-2 minutes is considered at the end of every 10 minutes walking test. For its complete evaluation, two types of walking experiments are performed, gait initiation for 3 minutes and gait assistance for 10 minutes. The gait assistance experiment is performed for 30 minutes, i.e. 3 sets of 10 minutes walking test.

\section{RESULTS AND DisCUSSIONS}

The proposed adaptive control strategy using the humanorthosis interaction torques has been tested and evaluated with five healthy individuals of the age group $37 \pm 9$, weight $80 \pm 8 \mathrm{~kg}$, and height $1.75 \pm 0.05 \mathrm{~m}$. The Institutions Ethical Review Board approved all experimental procedures involving human subjects. The results section has been divided into two parts in order to explain the gait initiation and the gait assistance scenarios.

\section{A. Gait Initiation}

The gait initiation strategy is evaluated by monitoring the deviation in the knee joint movement of the users, along with the interaction torques. The flexion and extension movement of the knee joint is monitored to differentiate the user's intention from a tremor movement. As shown in fig. 2, the user's right leg showed the gait movement initiation in most of the trials. This is also evident by the shift between the interaction torques of the right and left leg, both in hip and knee joint. The initiation of the gait is characterized by the flexion movement of the knee joint. For instance, at time 97 seconds the right knee joint shows a little displacement in the movement which initiates the gait cycle. The hip joint trajectory appears after a few seconds, immediately followed by the transition to the left leg. The right knee trajectory is slightly different because of the impulsive movement of the user.
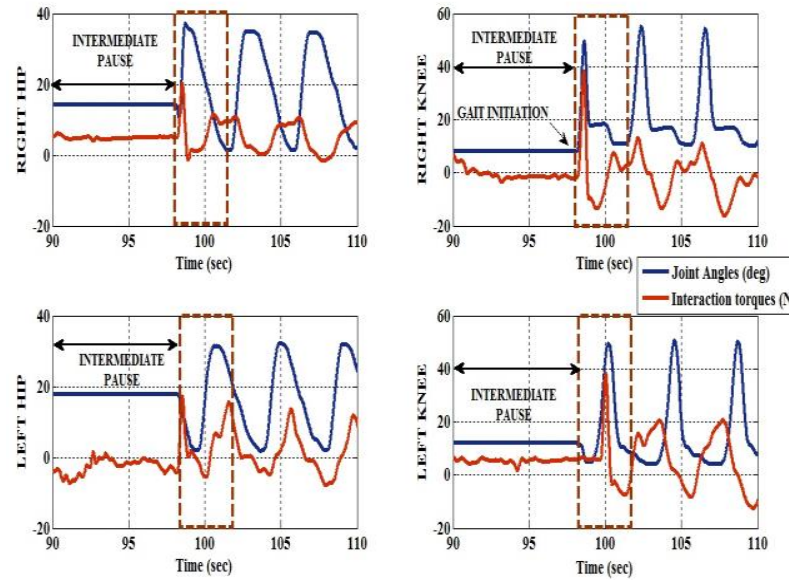

Figure 2. Gait initiation sequence of a healthy user

\section{B. Gait Assistance}

The efficiency of the adaptive assistance provided by the control model is evaluated in comparison with the reference gait pattern. The subjects performed a free normal walking movement with no restrictions on angle positions. The

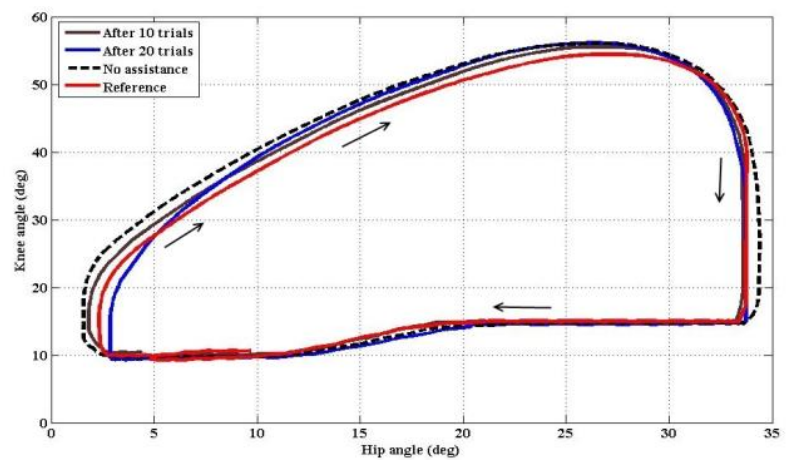

Figure 3. Changes in gait pattern of a healthy subject due to the effect of adaptive stiffness: Hip (deg) - Knee (deg)

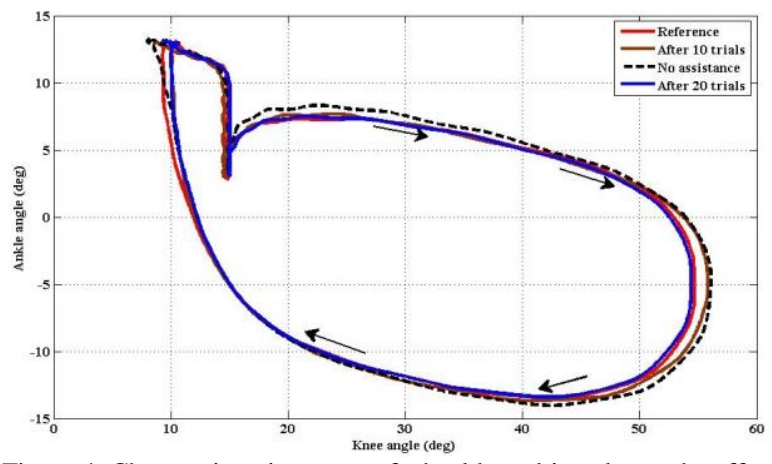

Figure 4. Changes in gait pattern of a healthy subject due to the effect of adaptive stiffness: Knee (deg) - Ankle (deg) 
deviation from the desired trajectory was found to be high in the case of free walking. After a series of trials (10) this error decreased gradually due to the effect of the stiffness acting on the joints. The stiffness variation helped to maintain this error within a specified range and following a similar pattern of incrementing and decrementing $\mathrm{K}$ at every joint.

Figures 3 and 4 show the gait performance of a healthy user demonstrating the influence of the stiffness variation proposed in this work. The initial walking with low stiffness value is presented as the 'no-assistance' mode. In comparison with the reference pattern, the no assisted walking is found to produce a maximum deviation. After the application of a variable stiffness, the user is able to walk within the predefined error limits. This also ensures that the user is not forced to follow the predefined trajectory The stiffness variation also converges with respect to the movement at the end of 10 trials. At the end of 20 trials, the user is following a movement which is quite similar to the reference pattern. The error was found to be within the defined limits for all the users and the interaction torques are bounded within the limits even in the presence of maximum stiffness.

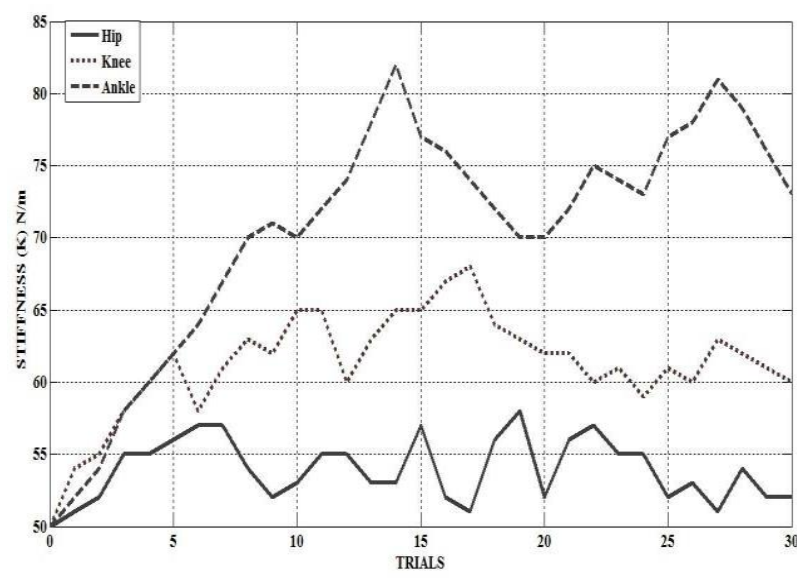

Figure 5. Stiffness variation of a healthy subject

The flexion and extension movement of the knee joint is essential in walking for maintaining the transition between gait phases. Thus the stiffness variation for the knee joint was observed to converge, because of the repetitive movements, after a few gait trials, as shown in fig. 5. In case of the ankle joint the stiffness behavior shows a different pattern due to the compensation of ground reaction forces. In the ankle joint, the deviation from the reference position is found to be higher, which explains the pattern of stiffness variation.

The confidence factor is used to act on the joint gradually, by varying its stiffness. The consequence is the relax intervals that appear as negative slope (decreasing stiffness). Low confidence factors will result in few and shorter steps of stiffness variation, so the increment will be faster. On the contrary, a high confidence factor will limit the increase of stiffness. The gradual increase in the stiffness value is due to the permanent difference in position error. The error in position of the joint in combination with the change in interaction torques results in a high stiffness value.

\section{CONCLUSION}

A compliance gait assistance triggered by user intention has been proposed and evaluated using a $\mathrm{H} 1$ exoskeleton with the aim of ensuring an effective and safe therapy. Both position error and human-orthosis interaction torques have been used as inputs. The gait initiation is determined at the beginning of each gait cycle from the input orders received from the user. Similarly, the stiffness value of each joint adapts dynamically to the user needs and keeps the position error bounded within the specified limits in real time. The wearable robot was tested with no body weight compensation neither treadmill training, which demonstrates the reliability of the control strategy in terms of ensuring dynamic stability in presence of ground reaction forces.

The experimental results showed that the user's gait intention was observed effectively without delay and followed by the leg movement. The results demonstrated that the evolution of the stiffness value does not follow a similar pattern for all the joints. The stiffness value converges to a value within a given range after a series of trials. This shows the efficiency of the proposed method for a real time process involving multiple joints.

\section{REFERENCES}

[1] H. Kazerooni, J. L. Racine, L. Huang and R. Steger, "On the control of the Berkeley lower extremity exoskeleton (BLEEX)," in IEEE International Conference on Robotics and Automation, ICRA, 2005.

[2] W. van Dijk and H. van der Kooij, "XPED2: A passive exoskeleton with artificial tendons," IEEE Robotics and Automation Magazine, pp. 56-61, December 2014.

[3] K. A. Strausser and H. Kazerooni, "The development and testing of a human machine interface for a mobile medical exoskeleton," in IEEE/RSJ International Conference on Intelligent Robots and Systems, San Francisco, CA, USA, 2011.

[4] M. Talaty, A. Esquenazi and J. E. Briceño, "Differentiating ability in users of the ReWalk powered exoskeleton," in IEEE International Conference on Rehabilitation Robotics, Seattle, USA, 2013.

[5] T. Yan, M. Cempini, C. M. Oddo and N. Vitiello, "Review of assistive strategies in powered lower-limb orthoses and exoskeletons," Robotics and Autonomous Systems, vol. 64, pp. 120-136, 2015.

[6] D. Novak, P. Rebersek, S. M. Maria De Rossi, M. Donati, J. Podobnik, T. Beravs, T. Lenzi, N. Vitiello, M. C. Carrozza and M. Munih, "Automated detection of gait initiation and termination using wearable sensors," Medical Engineering and Physics, vol. 35, pp. 1713-1720, 2013.

[7] W. Hasani, S. Mohammed, H. Rifaï and Y. Amirat, "Powered orthosis for lower limb movements assistance and rehabilitation," Control Engineering Practice, vol. 26, pp. 245-253, 2014.

[8] T. Noda, N. Sugimoto, J. Furukawa, M.-a. Sato, S.-H. Hyon and J. Morimoto, "Brain-Controlled Exoskeleton Robot for BMI Rehabilitation," in 12th IEEE RAS International Conference on Humanoid Robots, Osaka, Japan, 2012.

[9] S. Wang and e. al, "Design and Control of the MINDWALKER exoskeleton," IEEE Transactions on Neural Systems and Rehabilitation Engineering, vol. 23, no. 2, pp. 277-286, 2015.

[10] M. Aach, O. Cruciger, M. Sczesny-Kaiser, O. Höffken, R. C. Meindl, M. Tegenthoff, P. Schwenkreis, Y. Sankai and T. A. Schildhauer, "Voluntary driven exoskeleton as a new tool for rehabilitation in chronic spinal cord injury: a pilot study," The Spine Journal, vol. 14, pp. 2847-2853, 2014.

[11] L. Marchal-Crespo and D. Reinkensmeyer, "Review of control strategies for robotic movement training after neurologic injury," Journal of NeuroEngineering and Rehabilitation, vol. 6, no. 20, 2009.

[12] M. Bortole, A. J. Del-Ama, E. Rocon, J. C. Moreno, F. Brunetti and J. L. Pons, "A Robotic Exoskeleton for Overground Gait Rehabilitation," in IEEE International Conference on Robotics and Automation, Karlsruhe, Germany, 2013. 